\title{
Prevalence of and risk factors for coccidiosis in kiwi between 1977 and 2011
}

\author{
KJ Morgan ${ }^{\S *}$, KJ Morgan ${ }^{* \S}$, I Castro $^{\dagger}$, N Lopez-Villalobos*, WE Pomroy ${ }^{*}$, MR Alley", \\ BD Gartrell", S Hunter" and L Howe*
}

${ }^{*}$ Institute of Veterinary, Animal and Biomedical Sciences, Massey University, Palmerston North, New Zealand 4410 tInstitute of Agriculture and Environment, Massey University, Palmerston North, New Zealand, 4410

sAuthor for correspondence. Email: K.J.Morgan@massey.ac.nz

Supplementary Table 1. Details of 47 cases of coccidiosis affecting kiwi diagnosed by histological examination of post-mortem samples from kiwi submitted to the National Wildlife Mortality Database of New Zealand (Huia) between February 1977 and May 2011.

\begin{tabular}{|c|c|c|c|c|c|c|c|c|c|c|c|}
\hline Huia ID & Age & Species & Habitat & Season & $\begin{array}{l}\text { COD } \\
\text { Cocc }\end{array}$ & $\begin{array}{l}\text { Enteric } \\
\text { cocc }\end{array}$ & $\begin{array}{l}\text { Renal } \\
\text { cocc }\end{array}$ & $\begin{array}{l}\text { Hepatic } \\
\text { cocc }\end{array}$ & $\begin{array}{l}\text { Pulmona } \\
\text { ry cocc }\end{array}$ & $\begin{array}{l}\text { Splenic } \\
\text { cocc }\end{array}$ & $\begin{array}{l}\text { Oocysts } \\
\text { per gram }^{\text {a }}\end{array}$ \\
\hline 486 & $A$ & Brown & Captive & Winter & No & Yes & No & No & No & ND & small number \\
\hline 542 & $J$ & Brown & Captive & Summer & Yes & No & Yes & ND & ND & No & ND \\
\hline 576 & $J$ & Brown & Captive & Unknown & Yes & No & Yes & No & No & ND & ND \\
\hline 665 & $J$ & Brown & Captive & Spring & No & Yes & No & Yes & No & ND & ND \\
\hline 666 & $J$ & Brown & Captive & Summer & No & Yes & Yes & No & No & ND & ND \\
\hline 673 & $U$ & Brown & Captive & Winter & No & No & ND & No & ND & No & ++ \\
\hline 1432 & $J$ & Brown & Wild & Summer & No & Yes & Yes & Yes & No & No & 0 \\
\hline 1570 & $J$ & Brown & Captive & Autumn & No & Yes & Yes & No & ND & No & ND \\
\hline 1581 & $J$ & Brown & Wild & Autumn & No & No & Yes & No & No & No & ND \\
\hline 1913 & $J$ & Brown & Wild & Autumn & Yes & Yes & No & Yes & No & No & ND \\
\hline 1932 & $J$ & Brown & Wild & Autumn & No & ND & Yes & No & No & No & ND \\
\hline 1936 & $J$ & Brown & Crèche & Autumn & No & Yes & Yes & No & No & ND & ND \\
\hline 1976 & $C$ & Brown & Captive & Autumn & Yes & Yes & Yes & No & No & ND & 45,000 \\
\hline 2197 & $J$ & Brown & Captive & Spring & No & Yes & Yes & Yes & No & No & ND \\
\hline 2202 & $J$ & Brown & Captive & Spring & No & Yes & Yes & Yes & No & No & ND \\
\hline 2219 & $J$ & Brown & Wild & Spring & No & ND & Yes & No & No & ND & ND \\
\hline 2341 & A & Brown & Captive & Winter & No & Yes & No & No & No & No & ND \\
\hline 2520 & $J$ & Brown & Captive & Autumn & Yes & Yes & Yes & No & No & No & 1,800 \\
\hline 2586 & $J$ & Brown & Captive & Autumn & Yes & Yes & Yes & Yes & No & No & ND \\
\hline 2589 & $J$ & Brown & Captive & Autumn & Yes & Yes & Yes & Yes & No & Yes & ND \\
\hline 2598 & $J$ & Brown & Crèche & Autumn & No & ND & Yes & No & No & No & 0 \\
\hline 2612 & $J$ & Brown & Captive & Winter & No & No & No & No & No & No & 197 \\
\hline 2688 & $J$ & Brown & Crèche & Winter & Yes & Yes & No & No & No & No & 41,100 \\
\hline 2796 & $J$ & Brown & Captive & Spring & No & Yes & No & ND & No & ND & 0 \\
\hline 2853 & $J$ & Brown & Captive & Spring & No & Yes & No & No & No & No & 0 \\
\hline 3090 & $J$ & Brown & Captive & Autumn & No & Yes & Yes & No & No & No & ND \\
\hline 3101 & $J$ & Brown & Wild & Autumn & No & No & Yes & No & No & ND & 0 \\
\hline 3226 & $A$ & Brown & Wild & Winter & No & Yes & No & No & No & No & ND \\
\hline 3275 & C & Brown & Captive & Spring & No & ND & Yes & No & No & No & ND \\
\hline 3298 & $A$ & Brown & Captive & Spring & Yes & Yes & No & Yes & No & ND & ND \\
\hline 3321 & $J$ & Brown & Crèche & Spring & No & No & No & No & No & ND & 650 \\
\hline 3465 & $J$ & Brown & Wild & Unknown & No & Yes & No & No & No & ND & ND \\
\hline 3469 & $J$ & Brown & Wild & Summer & No & ND & ND & No & ND & ND & 57 \\
\hline 4444 & $A$ & Brown & Captive & Autumn & No & ND & No & Yes & No & No & ND \\
\hline
\end{tabular}

The content of this supplementary information has not been edited. All risk and liability rest with the authors. 


\section{Supplementary Information}

Morgan et al.

New Zealand Veterinary Journal:/ / dx.doi.org/10.1080/00480169.2014.932657

S2

\begin{tabular}{llllllllllll}
\hline Huia ID & Age & Species & Habitat & Season & $\begin{array}{l}\text { COD } \\
\text { Cocc }\end{array}$ & $\begin{array}{l}\text { Enteric } \\
\text { cocc }\end{array}$ & $\begin{array}{l}\text { Renal } \\
\text { cocc }\end{array}$ & $\begin{array}{l}\text { Hepatic } \\
\text { cocc }\end{array}$ & $\begin{array}{l}\text { Pulmona } \\
\text { ry cocc }\end{array}$ & $\begin{array}{l}\text { Splenic } \\
\text { cocc }\end{array}$ & $\begin{array}{l}\text { Oocysts } \\
\text { per gram }\end{array}$ \\
\hline 4467 & J & Tokoeka & Captive & Autumn & Yes & Yes & Yes & No & No & No & ND \\
4479 & A & Brown & Captive & Winter & No & ND & No & Yes & No & No & ND \\
4482 & J & Brown & Wild & Winter & No & ND & No & No & No & ND & 456 \\
4635 & J & Brown & Wild & Spring & Yes & No & No & No & No & No & 78,300 \\
4834 & A & Brown & Wild & Autumn & No & No & No & No & No & No & 4,350 \\
4846 & J & GSK & Captive & Winter & No & No & Yes & No & No & No & ND \\
4922 & J & Brown & Captive & Spring & No & Yes & No & No & No & No & ND \\
4929 & J & Brown & Captive & Winter & No & No & No & No & No & Yes & ND \\
5076 & J & Brown & Wild & Summer & No & Yes & No & ND & No & ND & ND \\
5133 & J & Brown & Captive & Autumn & Yes & Yes & No & Yes & Yes & No & 0 (wet smear) \\
5154 & J & Brown & Captive & Autumn & No & Yes & No & No & No & No & ND \\
6040 & A & Rowi & Wild & Autumn & No & Yes & ND & ND & ND & ND & 900 \\
6076 & J & GSK & Wild & Autumn & No & ND & Yes & No & No & No & 400 \\
\hline
\end{tabular}

${ }^{a}$ Results were as recorded by pathologist at necropsy, where available

$\mathrm{A}=$ adult; $\mathrm{C}=$ chick; $\mathrm{Cocc}=$ coccidiosis; $\mathrm{COD}=$ cause of death; $\mathrm{J}=$ juvenile; $\mathrm{ND}=$ not evaluated; $U=$ unknown. 